\title{
Excesso de peso e fatores associados em adolescentes
}

\author{
Excess weight and associated \\ factors in adolescents
}

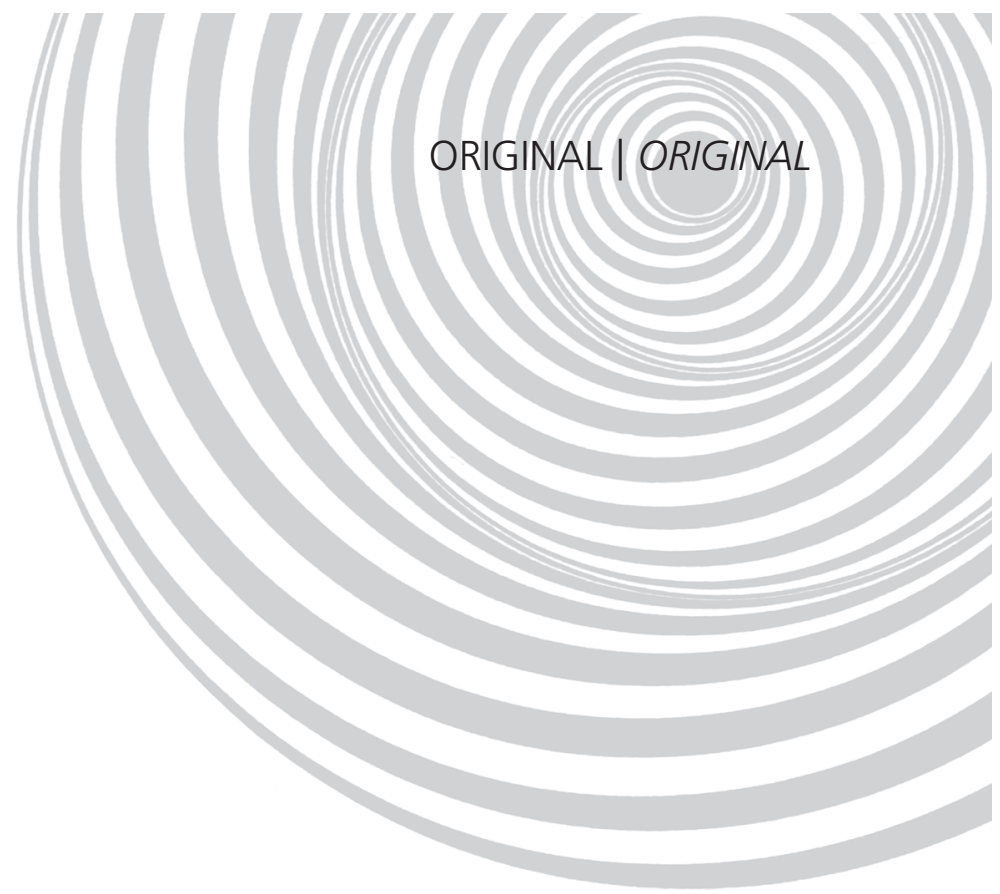

Edson dos Santos FARIAS'

Angelita Pereira dos SANTOS

José Cazuza de FARIAS-JÚNIOR²

Carlos Roberto Teixeira FERREIRA ${ }^{1}$

Wellington Roberto Gomes de CARVALHO3

Ezequiel Moreira GONÇALVES²

Gil GUERRA-JÚNIOR²

RE S U M O

\section{Objetivo}

Verificar a prevalência e os fatores associados ao excesso de peso em estudantes adolescentes em Rio Branco, Acre, Brasil.

\section{Métodos}

Estudo transversal com 741 adolescentes de escolas particulares de Rio Branco, Acre. Foram mensuradas as seguintes variáveis: sexo, idade, classe socioeconômica, escolaridade dos pais, número de moradores no domicílio, número de irmãos, atividade física, tempo de TV e de computador. A regressão logística binária foi utilizada para verficar as possíveis relações entre excesso de peso corporal e algus fatores associados.

\section{Resultados}

A prevalência geral de excesso de peso foi de $26,9 \%$ (30,0\% nos meninos e $24,2 \%$ nas meninas): apenas o sexo (masculino, $O R=1,34$ ), a classe socioeconômica (média alta, $O R=2,06$ e alta, $O R=2,36$ ) e a atividade física (insuficientemente ativo, $\mathrm{OR}=2,75$ ) apresentaram maior risco para desenvolver excesso de peso.

\section{Conclusão}

Os estudantes adolescentes do sexo masculino, de classe socioeconômica elevada e que praticam pouca atividade física foram os que apresentaram maior risco de excesso de peso nessa região do Brasil.

Termos de indexação: Atividade física. Classe socioeconômica. Obesidade. Sexo. Sobrepeso.

1 Universidade Federal do Acre, Centro de Ciências da Saúde e do Desporto. Rio Branco, AC, Brasil.

2 Universidade Estadual de Campinas, Faculdade de Ciências Médicas, Departamento de Pediatria. Cidade Universitária, 13083-970, Campinas, SP, Brasil. Correspondência para/Correspondence to: G GUERRA-JÚNIOR. E-mail: <gilguer@fcm.unicamp.br>.

3 Instituto Federal de Educação Ciência e Tecnologia do Sul de Minas Gerais, Centro de Ciências Aplicadas à Educação e Saúde, Curso de Educação Física. Muzambinho, MG, Brasil. 


\section{A B S T R A C T}

\section{Objective}

This study verified the prevalence of excess weight and associated factors in adolescents from private schools in Rio Branco, Acre, Brazil.

\section{Methods}

This cross-sectional study included 741 adolescents from private schools in Rio Branco, Acre. The following data were collected: sex, age, socioeconomic status, parents' education level, number of people in the household, number of siblings, level of physical activity, and number of hours spent daily watching television and using a computer. Logistic regression was used to investigate possible relationships between excess weight and associated factors.

\section{Results}

The general prevalence of excess weight was $26.9 \%$ (30.0\% for males and $24.2 \%$ for females). Inactive (OR=2.75) males $(O R=1.34)$ from the upper middle class $(O R=2.06)$ or upper class $(O R=2.36)$ were at highest risk of excess weight.

\section{Conclusion}

Inactive male adolescents belonging to the upper middle and upper classes are at high risk of excess weight in this region of Brazil.

Indexing terms: Physical activity. Socioeconomic status. Obesity. Sex. Overweight.

\section{N T R O D U Ç Ã O}

Diversos fatores estão associados ao desencadeamento da obesidade, como os genéticos, os fisiológicos e os metabólicos ${ }^{1}$. No entanto, aspectos do estilo de vida das pessoas, como inatividade física e padrão alimentar inadequado ${ }^{2}$, podem explicar o crescente aumento do número de adolescentes com excesso de peso ${ }^{3}$. A obesidade encontra-se associada a várias doenças crônicas, como diabetes Mellitus tipo 2, doenças cardiovasculares, hipertensão, dislipidemia, síndrome metabólica, osteoartrite e resistência à insulina ${ }^{4}$. Essas comorbidades, associadas a uma taxa reduzida de sucesso no tratamento desse problema de saúde, têm sido responsáveis por uma grande parcela dos gastos em saúde, e, por isso, a obesidade é considerada um dos maiores problemas de saúde pública.

Estudos nacionais e internacionais apontaram elevada prevalência de excesso de peso em adolescentes, com tendência de crescimento ${ }^{2,5-7}$. Os últimos levantamentos nacionais sobre excesso de peso em adolescentes indicaram que, em 30 anos, essa prevalência praticamente quadripli$\mathrm{cou}^{8}$. Esses resultados são similares aos que têm sido descritos em estudos com adolescentes de diversos países ${ }^{6,7}$.

A adolescência representa um período crítico em relação ao excesso de peso corporal, devido ao rápido crescimento linear, alterações hormonais, cognitivas e emocionais, que podem influenciar a quantidade de gordura corporal ${ }^{9}$. Adolescentes que apresentam excesso de peso tendem a ser adultos obesos ${ }^{10,11}$. Desse modo, a avaliação do estado nutricional de adolescentes tem sido recomendada, permitindo identificar os subgrupos com maior frequência de exposição a esse desfecho, que poderão ser alvo de intervenções para avaliação dos níveis de saúde da população jovem. Assim sendo, o objetivo deste estudo foi verificar a prevalência e os fatores associados ao excesso de peso corporal em estudantes adolescentes em Rio Branco (AC), Brasil.

\section{M É T O D O S}

Trata-se de um estudo descritivo e transversal de base escolar, realizado no segundo semestre de 2009, com estudantes adolescentes do ensino médio da rede particular de ensino da 
cidade de Rio Branco, estado do Acre, região Norte, localizado a sudoeste da Amazônia, Brasil.

A população total estimada de estudantes de ensino médio de Rio Branco (AC) em 2009 foi de aproximadamente 22 mil alunos (Secretaria Estadual de Educação - Setor de Estatística). Desses, 1747 (cerca de 8\% do total de estudantes de ensino médio) estavam distribuídos em 11 escolas particulares, e encontravam-se na faixa etária entre 14 e 18 anos, e eram de ambos os sexos.

O cálculo para determinar o tamanho da amostra baseou-se em uma prevalência estimada de $22 \%$ de excesso de peso (sobrepeso mais obesidade) $)^{12}$, erro amostral de três pontos percentuais, nível de confiança de $95 \%$, e população estimada de 1747 estudantes. Com base nesses parâmetros, o tamanho mínimo da amostra foi estimado em 516 estudantes. Considerando-se possíveis perdas e recusas, a esse valor foi acrescido $40 \%$, sendo o estudo realizado com 741 estudantes.

O processo de seleção dos alunos ocorreu em dois estágios: inicialmente realizou-se uma amostragem estratificada proporcional ao número de alunos de cada escola, e depois efetuou-se uma amostragem aleatória dentro de cada escola, incluindo-se no estudo todas as escolas urbanas públicas do município. Esse processo amostral permitiu que cada escolar tivesse probabilidade igual de ser sorteado, com reposição das perdas de até $40 \%^{13}$.

As variáveis independentes foram sexo, idade (em anos, centesimal), nível de escolaridade do pai, número de moradores no domicílio, número de irmãos, tempo assistindo televisão ( $\leq 2$ horas, $2-4$ horas e $\geq 4$ horas), tempo em frente ao computador ( $\leq 2$ horas, $2-4$ horas e $\geq 4$ horas), e classe socioeconômica ${ }^{14}$. Essa metodologia classifica as pessoas em classe econômica alta, média alta e média. Para estimar os níveis de atividade física, foi utilizado um questionário Internacional de Atividade Física (IPAQ), versão curta validado no Brasil por Matsudo et al..$^{15}$ e sua validade e reprodutibilidade para adolescentes foi determinada por Guedes et al. ${ }^{16}$. Para classificação do nível de atividade física foi adotado o seguinte critério: insuficientemente ativo (atividade leve com pouco esforço físico), moderadamente ativo (atividade moderada que precisa de esforço físico prolongado) e muito ativo (atividades de grande esforço físico).

O procedimento para aplicação dos questionários socioeconômico e de atividade física foi o mesmo, com a presença em sala de aula do pesquisador e do professor de sala que o auxiliou. Os questionários foram lidos e explicados pelo entrevistador, e, no decorrer do seu preenchimento, as dúvidas foram sendo sanadas. Após o preenchimento, os questionários foram recolhidos para análise. As perguntas de ambos os questionários foram todas fechadas.

Com base nas medidas de peso e estatura corporal, determinou-se o Índice de Massa Corporal $(\mathrm{IMC})$ dos estudantes $(\mathrm{IMC})=$ peso $(\mathrm{kg}) /$ estatura $\left(\mathrm{m}^{2}\right)$. A classificação do estado nutricional dos adolescentes, a partir do IMC, teve como base critérios propostos pela Center for Health Statistics in collaboration with the National Center for Chronic Disease Prevention and Health Promotion $(C D C)^{17}$. Os pontos de corte adotados foram: normal se $z \mid M C \leq 1,0$, e excesso de peso (sobrepeso mais obesidade), se $z \mid M C>1,0$; sendo sobrepeso quando $\mathrm{zIMC}>1,0$ e $\leq 2,0$, e obesidade quando $>2,0$.

Os dados foram analisados no Statistical Package for the Social Sciences 16.0, com um nível de significância de 5\%. O teste do Qui-quadrado para heterogeneidade (variáveis categóricas) e de tendência linear (variáveis ordinais) foi utilizado para comparar a prevalência de excesso de peso entre as categorias das variáveis independentes. Para avaliar a associação bruta e ajustada entre excesso de peso (baixo peso/peso normal $=0$ e sobrepeso/obesidade $=1$ ) com variáveis demográficas, socioeconômicas e comportamentais, utilizou-se a regressão logística binária. A significância das variáveis do modelo foi avaliada pelo teste de Wald para heterogeneidade e tendência linear, quando adequado. Foram consi- 
deradas para elaboração do modelo multivariável as variáveis com valor $p<0,20$ na análise bruta, sendo todas mantidas no modelo final.

A coleta de dados foi realizada mediante a autorização das direções das escolas, pais e alunos, através da assinatura do termo de consentimento livre esclarecido. O estudo foi aprovado pelo Comitê de Ética em Pesquisa com seres humanos da Universidade Federal do Acre (UFAC) (protocolo n²3107.009169/2009-89).

\section{RES U LTA D O S}

A amostra foi composta por 741 estudantes adolescentes de 14 a 18 anos de idade, sendo $54,1 \%(n=401)$ do sexo feminino. A maioria pertencia à classe socioeconômica média alta $(n=433,58,4 \%)$, era filho de pais com nível de escolaridade superior ( $n=473,63,8 \%$ ), com menos de três irmãos $(n=565,76,2 \%)$. Em relação ao nível de atividade física, 294 (39,7\%) adolescentes foram classificados como muito ativos, 296 (39,9\%) despenderam duas a quatro horas diárias à frente da TV e 290 (39,1\%) utilizaram o computador por tempo $<2 \mathrm{~h} / \mathrm{dia}$.

A prevalência geral de excesso de peso foi de $26,9 \%$ (sobrepeso $22,4 \%$ e obesidade $4,3 \%$ ). O sexo, a idade, a escolaridade dos pais, o número de moradores no domicílio e o número de irmãos não apresentaram associação com o excesso de peso (Tabela 1). No entanto, a classe socioeconômica e o número de horas assistindo TV (>4 horas) e utilizando computador ( $>4$ horas) apresentaram associação positiva e significativa com o excesso de peso corporal, enquanto que a atividade física (insuficientemente ativo) apresentou associação negativa e significativa (Tabela 1).

Na análise ajustada, mantiveram-se associados ao excesso de peso a classe socioeconômica e o nível de atividade física insuficientemente ativo. A variável sexo (masculino), que não havia se associado ao excesso de peso na análise bruta, passou a apresentar uma relação significativa com o excesso de peso (Tabela 2).

\section{I S C U S S Ã O}

Trata-se de um dos primeiros estudos a descrever a prevalência de excesso de peso corporal e analisar sua relação com fatores socioeconômicos e comportamentais em adolescentes que

Tabela 1. Prevalência de excesso de peso segundo sexo, faixa etária, classe econômica, escolaridade do chefe da família, número de moradores no domicílio, número de irmãos, atividade física, tempo de televisão e tempo de computador dos 741 estudantes adolescentes da rede particular de ensino da cidade de Rio Branco (AC), 2009.

\begin{tabular}{|c|c|c|c|}
\hline Variável & $n$ & $\%$ & $p$ \\
\hline Sexo & & & $0,075^{*}$ \\
\hline Masculino & 102 & 30,0 & \\
\hline Feminino & 97 & 24,2 & \\
\hline Faixa etária & & & $0,226^{*}$ \\
\hline 14-15 anos & 84 & 29,7 & \\
\hline 16-18 anos & 115 & 25,0 & \\
\hline Classe econômica & & & $0,028^{* *}$ \\
\hline Média & 12 & 15,4 & \\
\hline Média alta & 118 & 27,3 & \\
\hline Alta & 69 & 30,0 & \\
\hline Escolaridade do pai & & & $0,451^{* *}$ \\
\hline Fundamental & 15 & 21,1 & \\
\hline Médio & 55 & 27,9 & \\
\hline Superior & 129 & 27,3 & \\
\hline Número de moradores no domicílio & & & $0,314^{* *}$ \\
\hline$<3$ pessoas & 58 & 26,2 & \\
\hline 4-5 pessoas & 109 & 28,9 & \\
\hline$\geq 6$ pessoas & 32 & 22,4 & \\
\hline Número de irmãos & & & $0,684^{* *}$ \\
\hline$<3$ irmãos & 151 & 26,7 & \\
\hline 4-5 irmãos & 36 & 25,7 & \\
\hline$\geq 6$ irmãos & 12 & 33,3 & \\
\hline Atividade física & & & $<0,001^{* *}$ \\
\hline Insuficientemente ativo & 41 & 39,8 & \\
\hline Moderadamente ativo & 41 & 16,4 & \\
\hline Muito ativo & 117 & 20,8 & \\
\hline Tempo TV & & & $0,020^{* *}$ \\
\hline$<2 \mathrm{~h} / \mathrm{dia}$ & 59 & 25,3 & \\
\hline 2-4h/dia & 68 & 23,0 & \\
\hline$>4 \mathrm{~h} / \mathrm{dia}$ & 72 & 34,0 & \\
\hline Tempo computador & & & $0,003^{* *}$ \\
\hline$<2 \mathrm{~h} / \mathrm{dia}$ & 68 & 23,4 & \\
\hline 2-4h/dia & 58 & 23,4 & \\
\hline$>4 \mathrm{~h} / \mathrm{dia}$ & 73 & 36,0 & \\
\hline
\end{tabular}

*Teste do Qui-quadrado para heterogeneidade; ${ }^{\text {**}}$ Teste do Qui-quadrado para tendência linear. 
Tabela 2. Regressão logística múltipla e ajustada para excesso de peso e fatores associados em 741 adolescentes do ensino médio da rede particular da cidade de Rio Branco (AC), 2009.

\begin{tabular}{|c|c|c|c|c|c|c|}
\hline Variável & $\mathrm{OR}_{\mathrm{b}}$ & IC95\% & $p$ & $\mathrm{OR}_{\mathrm{a}}$ & IC95\% & $p$ \\
\hline \multicolumn{7}{|l|}{ Sexo } \\
\hline Masculino & 1,34 & $0,97-1,86$ & $0,076^{*}$ & 1,57 & $1,11-2,22$ & $0,011^{*}$ \\
\hline Feminino & 1 & & & 1 & & \\
\hline \multicolumn{7}{|l|}{ Classe econômica } \\
\hline Média & 1 & & $0,029^{* *}$ & 1 & & $0,022^{* *}$ \\
\hline Média alta & 2,06 & $1,08-3,95$ & & 1,97 & $1,00-3,89$ & \\
\hline Alta & 2,36 & $1,20-4,64$ & & 2,43 & $1,19-4,95$ & \\
\hline \multicolumn{7}{|l|}{ Atividade física } \\
\hline Insuficientemente ativo & 2,52 & $1,66-3,82$ & $<0,001^{* *}$ & 2,52 & $1,60-3,95$ & $<0,001^{* *}$ \\
\hline Moderadamente ativo & 0,75 & $0,46-1,21$ & & 0,78 & $0,48-1,28$ & \\
\hline Muito ativo & 1 & & & 1 & & \\
\hline
\end{tabular}

*Teste de Wald para heterogeneidade; ${ }^{* *}$ Teste de Wald para tendência linear. IC: Intervalo de Confiança.

residem na região Norte do Brasil. Os resultados deste estudo evidenciaram que a prevalência de excesso de peso foi elevada, sobretudo nos rapazes. Verificou-se, também, que os adolescentes mais ricos e aqueles fisicamente menos ativos tiveram maior chance de apresentar excesso de peso.

Em relação à prevalência de excesso de peso, os resultados do presente estudo indicaram que ela foi elevada e próxima da que tem sido descrita em outras investigações. Fernandes et al. ${ }^{18}$ encontraram em Presidente Prudente (SP) uma prevalência de excesso de peso em adolescentes de $28,6 \%$, e o estudo de Campos et al. ${ }^{19}$, feito em Fortaleza (CE), na região Nordeste, mostrou uma prevalência de sobrepeso/obesidade de $23,9 \%$, apontando para uma similaridade com os dados do presente estudo. Os estudos que envolveram adolescentes das regiões Norte, Nordeste e Sudeste do Brasil, de escolas particulares com melhor poder aquisitivo, apresentaram prevalência de excesso de peso similar, tendo a região Sul mostrado uma prevalência mais elevada12,20.

Um estudo feito na região Sul do País com estudantes adolescentes mostrou uma prevalência de excesso de peso de $39,2 \%$, sendo $27,9 \%$ para os meninos e $21,6 \%$ para as meninas. A prevalência na região Sul é maior que na região Norte, provavelmente por fatores como melhor dis- tribuição de renda, clima, etnia, hábitos alimentares etc. ${ }^{12}$.

Estudos internacionais conduzidos em países de economia emergente mostraram prevalência similar ao do presente estudo, como observado na Índia ${ }^{21}$, onde a prevalência de sobrepeso e obesidade foi respectivamente de 19,8\% e 9,5\%, enquanto que, em países desenvolvidos, a prevalência de excesso de peso tem sido acima de $30 \%$ em adolescentes ${ }^{22}$.

Para verificar possíveis influências dos pontos de corte adotados para classificar o estado nutricional dos adolescentes, utilizaram-se os critérios sugeridos pela World Health Organization (WHO). A aplicação desses critérios produziu uma prevalência de 27,1\%: bastante próxima da encontrada com o CDC-NCHS, que foi de $26,9 \%$.

A prevalência de excesso de peso no presente estudo foi maior nos meninos (30\%). Dados do levantamento realizado pelo Instituto Brasileiro de Geografia Estatístico ${ }^{23}$ mostraram que a parcela dos meninos de 10 a 19 anos de idade com excesso de peso passou de 3,7\% (1974-75) para 21,7\% (2008-09), já entre as meninas, o crescimento do excesso de peso foi de 7,6\% para $19,4 \%$. Outros estudos também observaram maior prevalência de excesso de peso entre os adolescentes do sexo masculino ${ }^{18,19}$. Estudo realizado no Reino Unido ${ }^{24}$ indicou que o excesso 
de peso é comum tanto em adolescentes do sexo masculino como do sexo feminino, todavia, na Itália, Finlândia e Áustria, a prevalência é mais elevada no sexo masculino; na Inglaterra e Espanha, resultados opostos são verificados ${ }^{25}$.

Conforme descrito na literatura $1,2,6,12,13,19$, no presente estudo foi observada maior prevalência de excesso de peso nos adolescentes que pertenciam aos estratos socioeconômicos mais elevados. Estudos revelam a existência de uma associação entre estudar na rede privada e apresentar maiores riscos de excesso de peso. Campos et al. ${ }^{19}$ encontraram uma prevalência de $23,9 \%$ em escolas particulares e 18,0\% em escolas públicas de Fortaleza (CE). Essa associação positiva entre excesso de peso e classe socioeconômica aparentemente é alicerçada no maior acesso por parte desses adolescentes a alimentos obesogênicos e em comportamentos sedentários ${ }^{25}$. No presente estudo, observou-se essa associação do excesso de peso com as classes socioeconômicas mais altas, corroborando assim outros estudos que indicam que adolescentes de escolas particulares e classes mais favorecidas têm maior prevalência de excesso de peso. Guimarães et al. ${ }^{26}$ verificaram que os estudantes cujos pais tinham escolaridade mais elevada tiveram chances 3,3 e 2,2 maiores de terem excesso de peso em relação aos de baixa escolaridade.

Quando analisada a associação entre atividade física e excesso de peso, foi observada uma associação inversa e significante, indicando que jovens fisicamente mais ativos tendem a apresentar menores chances de terem excesso de peso corporal. Esses resultados reforçam achados de estudos prévios. Estudos demonstraram que a frequência de atividade física dentro e fora da escola, em especial a atividade física de maior intensidade (exercícios vigorosos e intensos), aferidas por diferentes instrumentos, associaram-se inversamente a excesso de peso 20,27 .

No estudo conduzido por Olvera et al..$^{28}$, o aumento da intensidade de atividade física também se mostrou protetor para excesso de peso. Alguns estudos identificaram associação positiva entre praticar atividade física e excesso de peso ${ }^{28,29}$. Esses resultados reforçam a necessidade de estimular e apoiar a prática de atividade física em adolescentes, bem como a redução da exposição a comportamentos sedentários (tempo de TV, computador e games eletrônicos). Estudos recentes têm indicado que muitas crianças e adolescentes têm trocado as práticas de atividades físicas de intensidade moderada/vigorosa por atividades de baixa intensidade e, consequentemente, de baixo gasto energético, como assistir televisão, navegar na Internet, jogar videogame ou outros jogos eletrônicos durante as horas de lazer e tempo livre 24,25 .

Nesse sentido, maior atenção por parte dos órgãos de saúde pública, bem como estratégias de intervenção ainda na infância e adolescência devem ser iniciadas com a promoção de alimentação, atividade física e estilos de vida saudáveis, tanto no ambiente domiciliar como na escola, devendo ser os pais os maiores incentivadores à aderência da prática da atividade física e hábitos alimentares, uma vez que a literatura tem mostrado que crianças e adolescentes obesos apresentam maior risco de se tornarem adultos obesos e, consequentemente, maior probabilidade para desenvolvimento de doenças cardiovasculares $2,27,30$

Este estudo tem limitações que devem ser consideradas, como ter avaliado apenas os estudantes matriculados no ensino médio das escolas da rede particular de ensino: aspecto que pode representar um viés de seleção em potencial. Também, como limitação, pode ser considerado o método empregado para determinar a classe econômica dos adolescentes, que utiliza os utensílios do lar para classificação, implicando que certos utensílios, como aspirador de pó, não são muito utilizados nessa região do Brasil. Deve-se considerar também o fato de o aumento do poder de compra do salário-mínimo e o maior acesso ao crédito ter possibilitado a aquisição de utensílios por pessoas de menor condição sociocoeconômica, o que pode limitar a capacidade desse instrumento de classificar corretamente a classe econômica das pessoas. 
Conclui-se que a prevalência de excesso de peso nos estudantes adolescentes de Rio Branco (AC) foi elevada, com maior risco de exposição a esse desfecho no sexo masculino, na classe socioeconômica mais elevada e nos estudantes menos ativos fisicamente. Os resultados deste estudo podem auxiliar no planejamento de ações preventivas em relação ao excesso de peso em adolescentes. Estratégias que repercutam no controle e na manutenção do peso corporal - como redução do tempo diário assintindo à televisão ou no computador, melhora de hábitos alimentares e incentivo à aderência da prática da atividade física com maior participação nas aulas de educação física - podem ser fatores determinantes para efetividade das intervenções.

\section{COLABORADORES}

ES FARIAS participou na elaboração e na execução do estudo, na tabulação e na análise dos dados e nos resultados e na redação do artigo. JC FARIAS-JÚNIOR participou na tabulação dos dados, da análise estatística e da redação do artigo. AP SANTOS e CRT FERREIRA participaram da elaboração, da execução do estudo, e da redação do artigo. WRG CARVALHO e EM GONÇALVES participaram da análise dos resultados e da redação do artigo. G GUERRA-JÚNIOR participou da elaboração, da análise dos resultados, redação do artigo e supervisão do estudo.

\section{REFERÊ NCIAS}

1. Simony RS, Gimeno SGA, Ferreira SRG, Franco LJ. Prevalência de sobrepeso e obesidade em nipobrasileiros: comparação entre sexos e geração. Rev Nutr. 2008; 21(2):169-76. doi: 10.1590/s1415-52 732008000200005.

2. Rêgo ALV, Chiara VL. Nutrição e excesso de massa corporal: fatores de risco cardiovascular para adolescentes. Rev Nutr. 2006; 19(6):705-12. doi: 10.15 90/S1415-52732006000600007.

3. Toral N, Slater B, Silva MV. Consumo alimentar e excesso de peso em adolescentes de Piracicaba, São Paulo. Rev Nutr. 2007; 20(5):449-59. doi: 10.15 90/S1415-52732007000500001.
4. Cardoso LO, Engstron EM, Leite IC, Castro IRR. Fatores socioeconômicos, demográficos, ambientais e comportamentais associados ao excesso de peso em adolescentes: um revisão sistemática da literatura. Rev Bras Epidemiol. 2009; 12(3):378-403. doi: 10.1590/s1415-790x2009000300008.

5. Weil EJ, Curtis JM, Hanson RL, Knowler WC, Nelson $R G$. The impact of disadvantage on the devlopement and progression of diabetic kidney disease. Clin Nephrol. 2010; 74 (Suppl 1):s32-s8.

6. Beck CC, Loper AS, Giuliano ICB, Borgatto AF. Fatores de risco cardiovascular em adolescents de município do sul do Brasil: prevalência e associações com variáveis sociodemográficas. Rev Bras Epidemiol. 2011; 14(1):36-49. doi: 10.1590/s1415-7 90x2011000100004.

7. Goetzel RZ, Gibson TB, Short ME, Chu BC, Waddell $J$, Bowen J, et al. A multi-worksite analysis of the relationships among body mass index, medical utilization, and worker productivity. JOME. 52(Suppl 1):S52-S8. doi: 10.1097/JOM.obo13e318 1c95b 842010.

8. James PT, Leach R, Kalamara E, Shayeghi M. The Worldwide obesity epidemic. Obes Rev. 2001; 9(Suppl1):s228-s33. doi: 10.1111/j.1740-8709.2 009.00232.x/pdf

9. Deitel $M$. The international obesity task force and "globesity". Obse Surg. 2002; 12(5):613-4. doi: 10.1381/096089202321019558.

10. Pinto ICS, Arruda IKG, Diniz AS, Cavalcanti AMTS. Prevalência de excesso de peso e obesidade abdominal, segundo parâmetros antropométricas, e associação com maturação sexual em adolescentes escolares. Cad Saúde Pública. 2010; 26(9):1727-37. doi: 10.1590/s0102-311X2010000900006.

11. Yuca SA, Yilmaz C, Cesur $Y$, Dogan M, Kaya A, Basaranoglu M. Prevalence of overweight and obesity in children and aolescents in eastern Turkey. J Clin Res Pediatr Endocrinol. 2010; 2(4):159-63. doi: 10.4274/jcrpe.v2i4.159.

12. Suñe FR, Dias-da-Costa JS, Olinto MTA, Pattusi MP. Prevalência e fatores associados para sobrepeso e obesidade em escolares de uma cidade no sul do Brasil. Cad Saúde Pública. 2007; 23(6):1361-71. doi: 10.1590/s0102-311×2007000600011.

13. Vanzelli AS, Castro CT, Pinto MS, Passos SD. Prevalência de sobrepeso e obesidade em escolares da rede pública do município de Jundiaí, São Paulo. Rev Paul Pediatr. 2008; 26(1):48-53. doi: 10.1590/s 0103-05822008000100008.

14. Associação Nacional de Empresas de Pesquisa. Critério de classificação econômica [Internet]. 2010. [acesso 2010 mar 5]. Disponível em: <http:/ /www. anep.org.br/mural/anep/04-12-97-cceb.htm> . 
15. Matsudo S, Araújo T, Matsudo V, Andrade D, Andrade E, Oliveira LC, et al. Questionário Internacional de Atividade Física (IPAQ): estudo de validade e reprodutibilidade no Brasil. Rev Bras Ativ Física Saúde. 2001; 6(2):5-18.

16. Guedes DP, Lopes CC, Guedes JERP. Reprodutibilidade e validade do questionário internacional de atividade física para adolescentes. Rev Bras Med Esporte. 2005; 11(2):151-8. doi: 10.1590/\$1517-86 922005000200011.

17. Centers for Disease Control and Prevention. National Center for Health Statistics. 2000 CDC Growth Charts for the United States. Washington (DC): CDC; 2002 [cited 2010 Feb 20]. Available from: <http://www.cdc.gov/growthcharts>.

18. Fernandes RA, Codgno JS, Cardoso JR, Ronque ERV, Freitas Junior IF, Oliveira AR. Fatores associados ao excesso de peso entre adolescentes de diferentes redes de ensino do município de Presidente Prudente, São Paulo. Rev Bras Saúde Matern Infant. 2009; 9(4):443-9. doi: 10.1590/s1519-38292009 000400009.

19. Campos LA, Leite AJM, Almeida PC. Prevalência de sobrepeso e obesidade em adolescentes escolares do município de fortaleza, Brasil. Rev Bras Saúde Mater Infant. 2007; 7(2):183-90. doi: 10.15 90/s1519-38292007000200009.

20. Dutra $C L$, Araújo $C L$, Bertoldi $A D$. Prevalência de sobrepeso em adolescentes: um estudo de base populacional em uma cidade no sul do Brasil: Cad Saúde Pública. 2006; 22(1):151-62. doi: 10.1590/s01 02-311x2006000100016.

21. Kotian MS, Kumar G, Kotian S. Prevalence and determinants of overweight and obesity among adolescent school children fo south Karnataka, India. Assoc Comm Med. 2010; 35(1):176-8. doi: 10.4103/0970-0218.62587.

22. Dodor BA, Shelley MC, Hausafus CO. Adolescents health behaviors and obesity: does race affect this epidemic? Nutr Res Pract. 2010; 4(6):528-34. doi: 10.4162/mrp.2010.4.6.528.
23. Instituto Brasileiro de Geografia e Estatística. Pesquisa de orçamentos familiares 2008-2009: antropometria e estado nutricional de crianças, adolescentes e adultos no Brasil [Internet]. Rio de Janeiro; 2009 [acesso 2010 jun 5]. Disponível em: <http:// www.ibge.gov.br/presidenci/notiias/noticia_ visuliza.php?d_noticia=1699eid-pagina=1 > .

24. Reilly JJ. Obesity in childhood and adolescence: evidence based clinical and public health perspective. Postgrad Med J. 2006; 82:429-37. doi: 10.1136/ pgmj.2005.043836.

25. Foti K, Lowry R. Trends in perceived overweight status among overweight and noverweight adolescents. Arch Pediatr Adolesc Med. 2010; 164(7):634-42. doi: 10.1136/pgmj.2005.043836.

26. Guimarães LV, Barros MBA, Martins MSAS, Duarte EC. Fatores associados ao sobrepeso em escolares. Rev Nutr. 2006; 19(1):5-7. doi: 10.1590/s1415-52 732006000100001.

27. Baker JL, Olsen LW, Sorensen TI. A childhood body-mass index and the risk of coronary heart disease in adulthood. J Medical. 2007; 357(23): 2329-57. doi: 10.1056/NEJMoa072515.

28. Olvera N, Graham M, McLeod J, Stephanie FK, Butte NF. Prometing moderate-vigorous phisical activity in overweight minority girls. Int J Pediatr. 2010; 2010(2010):1-7. doi: 10.1155/2010/415123.

29. Farias ES, Paula F, Carvalho WRG, Gonçalves EM, Baldin AD, Guerra-Júnior G. Efeito da atividade física programada sobre a composição corporal em escolares adolescentes. J Pediatr. 2009, 85(1):28-34. doi: 10.2223/JPED.1864.

30. Enes CC, Slater B. Obesidade na adolescência e seus principais fatores determinantes. Rev Bras Epidemiol. 2010; 13(1):163-71. doi: 10.1590/s141 5-790x2010000100015.

Recebido em: 2/5/2011 Versão final em: 10/10/2011 Aprovado em: 10/1/2012 